\title{
Emergence of Protests During The COVID-19 Pandemic: Quantitative Models To Explore The Contributions of Societal Conditions
}

Koen van der Zwet ( $\nabla$ k.vanderzwet@uva.nl )

University of Amsterdam

Tom van Engers

University of Amsterdam

Ana Martins Botto de Barros

TNO

Peter Sloot

University of Amsterdam

\section{Article}

Keywords: pandemic, resistance, quantitative, dynamics

Posted Date: December 3rd, 2021

DOI: https://doi.org/10.21203/rs.3.rs-845904/v1

License: (9) This work is licensed under a Creative Commons Attribution 4.0 International License.

Read Full License

Version of Record: A version of this preprint was published at Humanities \& Social Sciences Communications on March 2nd, 2022. See the published version at https://doi.org/10.1057/s41599-02201082-y. 


\title{
Emergence of protests during the COVID-19 pandemic: quantitative models to explore the contributions of societal conditions
}

\begin{abstract}
The outbreak of the COVID-19 pandemic has led to a resurgence of protests. Various societal conditions of social systems, such as economic stability, demographic ageing, and political elites, are often associated to the emergence of civil resistance movements. Several qualitative and quantitative models have been developed to analyse the relationship between societal conditions and the emergence of protests. The existing models use the underlying assumptions that these conditions operate in similar time-scales. However, the analysis of social systems also shows the importance of considering explicitly the inherent time-scales particularly slow-fast dynamics. The sudden and dramatic disruptive force of the pandemic has yield fine-grained data sets that can be used to better grasp the different dynamics of this social phenom. This paper proposes an integrated approach to explore the relationship between societal conditions and the emergence of protests in the context of the COVID-19 pandemic. First, a literature based causal-loop-diagram is constructed to conceptualise the emergence of civil resistance as a result of intertwined dynamics. Based on the derived factors in the literature study, a data set is constructed to enable this analysis. Furthermore, by means of statistical and computational modelling we conduct a quantitative analysis in which we compare the emergence of protests for 27 countries during the pandemic. Also based on the factors found in literature we have constructed a system dynamics model that explicitly models the development of societal strains and social mobilisation in order to provide a better quantitative explanation of the emergence of protests. We found that while fast-changing factors are better estimators for 'when' civil resistance emerges, slow-changing factors are better estimators of 'how' civil resistance manifests itself in terms of the relative intensity of the protests in specific countries.
\end{abstract}

\section{Introduction}

Several civil resistance movements against the governmental institutions have emerged during the COVID-19 pandemic in 2020. These societal resistance movements are the result of citizens seeking to alter power deficits and effect social transformation by mobilising normal citizens for sustained political action ${ }^{1}$. Non-violent actions can take several forms, from which protests are very common. Globally, more than 25 significant protest movements have been directly related to the COVID-19 pandemic ${ }^{2}$. According to data of the Armed Conflict Location \& Event Data Project (ACLED), around 25\% of the protest events in 2020 can be linked to the COVID-19 pandemic (Figure 1). The emergence of civil resistance movements are common during the outbreak of epidemic diseases, as similar events were observed during the outbreak of EBOLA, SARS and MERS ${ }^{3}$.

In this paper, we propose approach for modelling the social dynamic of civil resistance emergence by explicitly considering socioeconomic conditions and social-psychological factors in the context of the COVID-19 pandemic. Along three phases, we model this phenomena on a conceptual, quantitative, and computational level. First we conduct a literature review to identify and analyse relevant factors for understanding the emergence of civil resistance and the disruptive effect of a pandemic. A causal-loop diagram (CLD) is constructed to summarise these findings and provide a conceptual understanding that brings together the different perspectives. Secondly, this model was applied in the context of the COVID-19 pandemic. Using data analysis we explored the impact of the identified factors empirically. By matching the factors with data proxies we create a data set to enable quantitative analysis of this phenomenon. The data enables analysis of the number of protests and riots on the daily-scale in terms of temporal resolution and country-level scale in terms of spatial resolution. Moreover, the variables also capture socioeconomic factors, disruptive effects by the pandemic and interventions. The effect of these variables upon civil resistance is compared in terms of explanatory value using statistical modelling. Lastly, we construct a system dynamics model (SDM) to analyse the interplay between the development of pressure and mobilisation of civil resistance. We try to model the intertwined underlying societal processes that drive the emergence of protest events. This modelling step provides several explanations of the potential, and vulnerability of using data on societal conditions to understand the emergence of civil resistance.

This paper contributes to the development of modelling the political stability of societies and to the study of the impact of disruptive events. Through modelling and analysis of data sets, we can analyse the interplay between societal dynamics, government interventions and the emergence of civil resistance movements at various time-scales. Most importantly, in the various modelling phases we evaluate the added value and limitations of data modelling and simulation for understanding civil resistance movements using currently available data sets and provide future directions. 


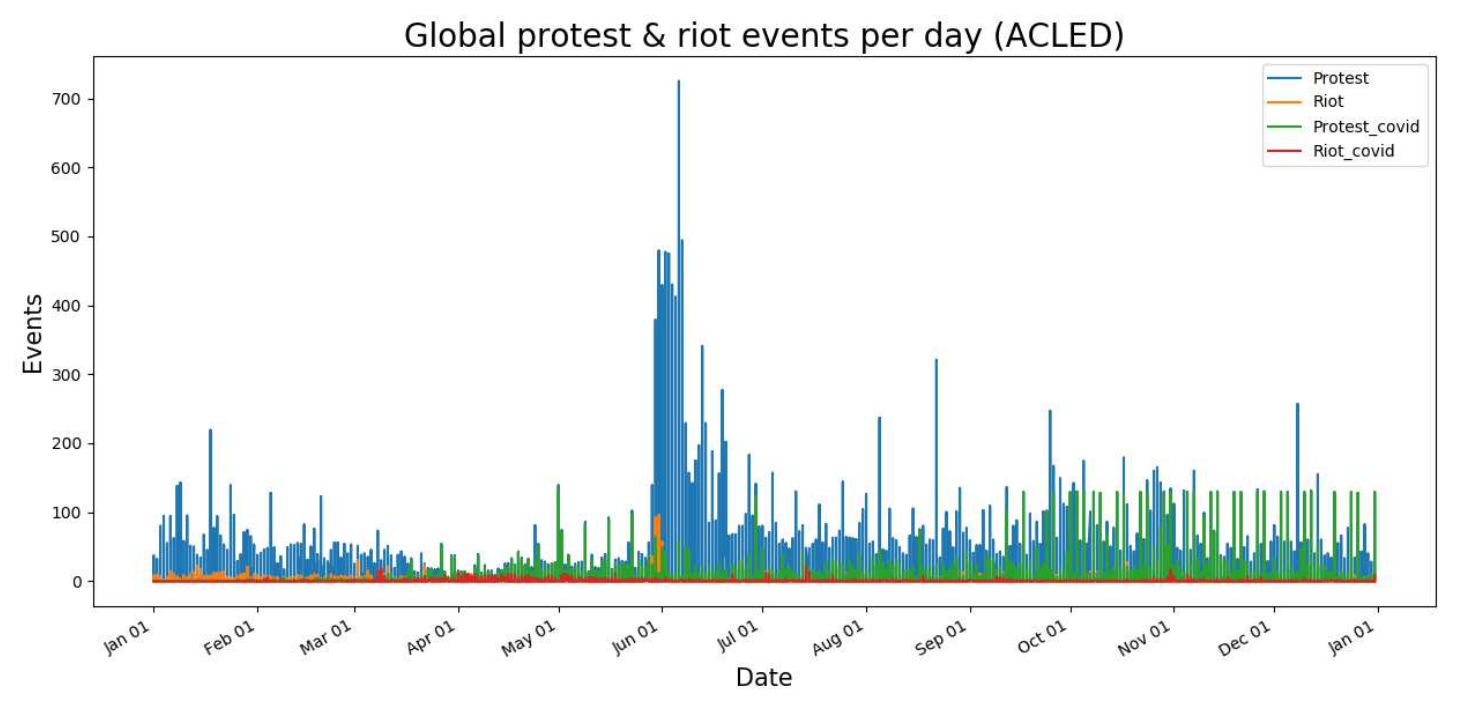

Figure 1. The number protest and riot events globally on a daily scale during the year 2020. (Data: ACLED)

\section{Civil resistance during COVID-19}

The current outbreak of COVID-19 has caused an increased uncertainty about health conditions and has yield unprecedented measures to constrain the spread of a disease. These developments test the resilience capacity of societies as they adapt in many ways to deal with the pandemic's undesired effects. The uncertainty about the effects of the disease and the chance to become infected causes fear amongst people ${ }^{3}$. Moreover, while containment measures intend to limit the spread of the disease through large-scale behavioural change, they induce psychological burdens on individuals ${ }^{4}$. On the level of an individual, these burdens are driving factors for the development of stress, uncertainty, anxiety, and anger ${ }^{5}$. On a societal level, these factors might instigate the development of social unrest in forms of widespread panic. The emergence of social unrest and the widespread uncertainty causes that people might fear that the containment measures are harmful rather than helpful ${ }^{5}$. Moreover, people tend to become more susceptible to conspiracy theories ${ }^{4}$.

The aforementioned effects cause or amplify the development of societal strains. Once the development of these strains overshoot or outpace the adaption capability, a society may become unstable and prone to conflict ${ }^{6}$. Societal disorder may trigger civil resistance movements or civil war. The occurrence of various deprived societal conditions such as poverty, state-led discrimination or a lack of civil liberties correlate with the onset of non-violent uprisings ${ }^{7-9}$. These conditions can be exploited by skilful activists to mobilise people to join civil resistance movements and spark the onset of protests, strikes or resistance in other forms.

Data models that analyse structural conditions have been unable to account for agency-oriented individual-level dynamics, such as strategic decisions ${ }^{8}$. This has limited the predictive power of current models that study the onset of civil resistance. Most importantly, these studies limit the study of the emergence of civil resistance to a yearly cross-national analysis. Specific societal conditions are assumed to be generalizable for all civil resistance movements. However, disruptive events, for example the outbreak of COVID-19, have a specific impacts on the societal stability and might trigger specific micro-interactions that yield civil resistance behaviour. Understanding how these specific effects percolate through the society might yield better quantitative models.

Moreover, predicting the onset civil resistance movements year-by-year assumes that all conditions change somewhat with similar pace. However, the structural factors affecting human attitudes and behaviours operate on different time-scales ${ }^{10}$. The most important aspect of analysing social systems is the understanding that these systems have multiple time-scales and are high-dimensional ${ }^{11}$. Demographic changes occur the slowest, political elites and economic stability vary at an intermediate pace, and individual emotions change very rapidly. As the time-scales on which these representations change differ, their impact on the adaptability of the society might differ. This can be hypothesised from the theory of downward causation. While 'collective-level' dynamics -such as economic stability- result from 'individual-level' interactions, individuals can use these 'coarse-grained' representations to make strategical decision ${ }^{12}$. For example, the increase of unemployment during the COVID-19 pandemic could be observed by skilled leaders of civil resistance movements and exploited to recruit civilians that 
are unemployed or are on the brink of losing their job. As various socioeconomic factors and political dynamics change at different time-scales, the society is requested to adapt differently. Therefore, some dynamics might predict 'where' the onset of civil resistance will take place, while others might explain 'when' and 'how'.

\section{The emergence of civil resistance and impact of epidemics}

A first step in understanding why civilians initiate and participate in civil resistance is the identification of various factors that influence this behaviour. These factors were identified through a literature review. First, an introduction of various theories of underlying dynamics of civil resistance will provide a structure of various societal dynamics. Along this structure, general and COVID-19 specific psychological, societal, political, economic, and health-care related factors are identified that might trigger civil resistance. These factors and their relationships are summarised and explored using a CLD.

Dissatisfaction, political opportunity, and mobilisation capacity are the three perspectives to explain the emergence of civil resistance movements ${ }^{13}$. At its core civil resistance movements consist of citizens that collectively attempt to impose a change in their living conditions by challenging a state opponent ${ }^{14}$. The idea that people are motivated to engage in resistance due to discontent over their living conditions is encapsulated by the theory of relative deprivation ${ }^{15}$. The existence of such feelings are usually described as grievances. These feelings can be shared among those in specific ethnological groups, which strengthens cohesion and propel polarisation and fragmentation.

State failure or fragility is the absence of state capacity to avert the development of these grievances. Civil resistance might emerge as the ethnological groups perceive a lack of power by the state authorities to impose change or do not feel represented by the state authority ${ }^{16}$. This centralises the role of the state in the development of civil resistance. An eroding state capacity opens the opportunity for violent civil resistance, as a weakened government is less able to suppress mobilisation ${ }^{17}$. These opportunities originate from changes in political structure, signaling effects or context specific issues ${ }^{18}$. The transition of power in a governmental structure, for example, could signal less stability and less strength of a regime or a possibility of enforce change.

These factors thus convince groups that either the chance of success of the rebellion is relatively high, or the risk of orchestrating rebellion is relatively low. This mobilisation phase is argued to be a specific decisive factor of whether rebellion occurs. Effective rebellion is dependent on the number of people, financial support, and other resources, which requires specific organisational skills and capacity. Additionally, mobilisation might be easier in a crowded urban area or when a large proportion of the population is relatively young ${ }^{19}$.

The dynamics of dissatisfaction, opportunity and mobilisation of civil resistance provides a categorisation of factors. In the next sections, we extract factors along each of these categories. Through a literature review we extract factors within these each of these categories. As these factors influence the civilians' decision to engage in civil resistance, they determine state stability. While some of these factors are very context dependent and fluid, others, such as demographic and economic situation, are quite structural and beyond the control of activists ${ }^{8}$. The various underlying factors, how they influence each other, and their stability, the time-scale on which they change, are explored in the context of COVID-19 outbreak.

\section{Pathways towards civil resistance}

The literature review shows that the emergence of civil resistance and strategic decisions seem to be influenced by many different societal factors and dynamics. In particular its dynamics are mainly driven by dissatisfaction, opportunity and mobilisation. Using these insights, we develop an integrated mapping of possible causal relationships between the identified factors to structure possible pathways towards civil resistance. A Causal Loop Diagram (CLD, see Figure 2.) is constructed along three driving dynamics -dissatisfaction, opportunity and mobilisation- triggered by epidemic outbreak. The resulting model brings together the different perspectives and underlying relations between the identified effects of societal factors and dynamics towards civil resistance. The spread of the epidemic disease challenges the stability of the society as it increases the development of grievances, lowers the government legitimacy in specific population segments, and creates fear and uncertainty. Moreover, possible interventions to contain the pandemic influence society and as such need to be explicitly modelled. An explanation of the causal links and reinforcing- and balancing loops identified is provided in the next sections.

\section{Dissatisfaction}

Unequal living conditions or unequal distribution of political power is the central motivation for people to engage in civil resistance $^{16}$. People may perceive a gap between their current situation and a desired minimum-level standard for specific needs. The prioritisation of these needs might be cultural dependent ${ }^{20}$. Moreover, the process of prioritisation might be not rational all the time and personality dependent. While the exact analysis of these needs is beyond the scope of this project, the vocalisation of these needs by socially active groups point to a connection between the psychological state of individuals, their behaviour and societal conditions. 


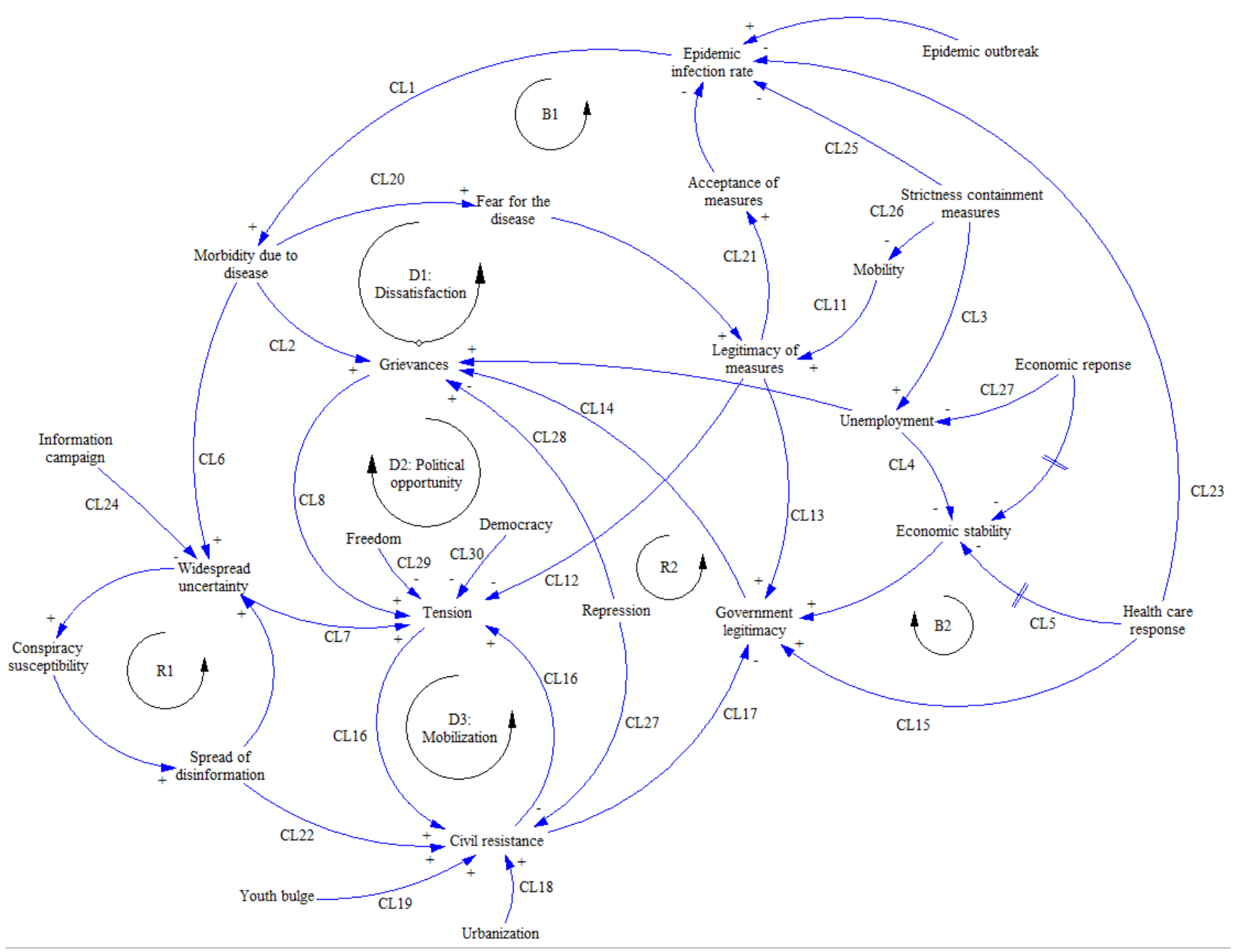

Figure 2. Causal-loop diagram (CLD) of the pathways towards civil resistance. Variables connected with arrows indicate causal links extracted from literature.

Living conditions are highly dependent on the level of safety that people can provide themselves and their relatives. Environments with high levels of violence, corruption, and discrimination endanger people's daily life. These conditions can possibly result from societal struggle for resources and power or from the actions of authorities, as factionalised elites can lead to state-led discrimination or repression of ethnographical population segments ${ }^{16}$.

The COVID-19 pandemic worsened global safety conditions directly, as infections pressured health care capacity and caused severe increase of morbidity (CL1). Poverty is the greatest risk factor in epidemics ${ }^{21}$. For example, the Ebola outbreak in Liberia was exacerbated by a lack of physical infrastructure, proper sanitation, and health facilities. Poverty among people causes an inability to access enough food, education and health care, which result in a high level of infant mortality and a low life expectancy. Poverty among people causes an inability to access enough food, education and health care, which result in a high level of infant mortality and a low life expectancy, thus increasing existing grievances (CL2).

Moreover, especially in states with limited state capacity, authorities are unable to trace the effects and spread of the disease and as such are at risk to lose control even further ${ }^{22}$. The economic stability of a state also highly impacts living conditions. High levels of unemployment and instable economies can push people into to poverty. The macro-economic impact of epidemics are severe as it lowers the demand rates $^{23}$. Mitigating measures such as quarantine lowers the productivity and

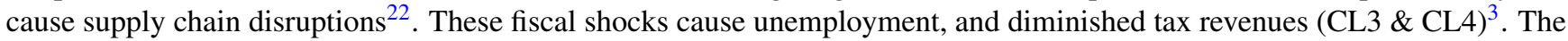
necessity to invest in health care related measures claims financial resources (CL5) ${ }^{22}$. Reports of a disease outbreak can already cause expectations and psychological sentiment to the extent that it will cause economic effects.

Additionally, pandemics are marked by many psychological stressors ${ }^{5}$. The widespread uncertainty, confusion and sense of urgency creates a societal tension (CL7) as individuals deal with a psychological burden, triggered by anxiety, anger and 
distress due to the pandemic (CL6). These developments can trigger individual's psychological traits such as behavioural immune system, coping styles, and beliefs which influence social attitudes and interactions ${ }^{5}$. The societal effects of these burdens can be immense. Due to uncertainty, people are more vulnerable to fake news and conspiracy theories, which reinforces the uncertainty $(\mathrm{R} 1)^{4}$. Groups can be stigmatised and blamed for the disease and its consequences ${ }^{3,21}$. This can lead to social tension (CL8), discrimination and eventually violence against minorities ${ }^{24}$.

\section{Opportunity}

Civil resistance does not happen in a vacuum, rather the goals, strategies, and tactics of activists are aimed to capitalise upon specific opportunities that are provided to them ${ }^{18}$. Political opportunity theory aims to understand the structure, political context, in which civil resistance happens. A window of opportunity for resistance can open up as specific developments favour the expected effect of protests, or if they are expected to be less costly ${ }^{25}$. During a political transition, for example, an opportunity can arise to push for reforming laws by the new incumbent ${ }^{7}$. In contrary, governments that are able to provide freedom (CL29) and political rights (CL30) are less likely to be confronted with an outbreak of civil resistance ${ }^{8}$.

During the pandemic, morbidity and social inequality may amplify social tensions due to grievances, as those with limited access to healthcare services, and economic security are effected most by epidemic diseases ${ }^{3,21}$. To mitigate the impact of the epidemic disease, governments are forced to take many unpopular containment measurements to lower mobility of citizens (CL11) (e.g. quarantine and curfews) to limit the spread of the disease. These issues can be politised and open political opportunities to decrease government legitimacy (CL12 \& CL13).

The uncertainty about the efficiency of these various containment strategies lowers the trust in government capacity ${ }^{5}$. Moreover, as the containment measurements have a large economic and societal impact, clashes between politicians can reduce the perceived legitimacy of the government to deal with the effects of the pandemic (CL14) ${ }^{3}$. In contrary, strict public-health measures imposed in the Netherlands during the severe phase of the COVID-19 pandemic actually increased the trust in the government $(\mathrm{CL} 15)^{26}$. Due to these tensions inadequate or conflicting information provision from government institutions can cause long-term effects of confusion and anger $(\mathrm{R} 2)^{27}$.

\section{Mobilisation}

The number of participants of a civil resistance movement influences its ability to gain political influence (CL17) ${ }^{1}$. Escalation from dissatisfaction to collective actions requires organisation and mobilisation (CL16) ${ }^{28}$. Mobilisation requires people to go through four processes from inactive to full participation ${ }^{29}$. First, people have to sympathise with a movement. Second, these people and have be targeted by the movement to convince them to join. Third, people have to motivated to actually participate in activities. Lastly, the people involved need to find effective manners to participate. Various roles -for example leaders, spreaders, and followers- within movements exist to facilitate these processes ${ }^{30}$. Specific factors can accelerate these processes $^{29}$. For example, social embeddedness enables people use their social network to influence others. This enables the processes of emotional contagion in which people converge emotionally and even develop the tendency to mimic and synchronise facial expressions, vocalisations and postures ${ }^{31}$.

The mobilisation process can also be facilitated by geographical factors such as urbanisation (CL18) or regional contagion of civil resistance movements ${ }^{8}$, or demographic factors such as the 'youth bulge' in which a population consists of a higher proportion of youth $(\mathrm{CL} 19)^{7}$. These circumstances can strengthen motivational and political opportunity factors. For example, although the rate of unemployment in Egypt changed little between 1990 and 2010, in 2010 half of all Egypt's unemployed belonged to the 20-24 age cohort. This group of about one million unemployed youths became the main striking force of the revolution ${ }^{10}$.

During pandemics, an increase of cohesion and politicised collective identity creates social embeddedness within specific groups in a society, which increases behavioural contagion and potentially mobilisation ${ }^{32}$. The direct and indirect effects of an epidemic disease causes the spread of fear (CL20) ${ }^{33}$. The spread of fear has various desired and undesired effects ${ }^{5}$. First, it causes that people are more cautious in their efforts to avoid infection (CL20). Second, people tend to minimise their social interactions which potentially is slowing down the mobilisation process (CL21) ${ }^{33}$. Lastly, fear tends to increase the widespread uncertainty in a society ${ }^{5}$. Next to the spread of fear, the unpopular character of countermeasures triggers a natural response by people to question government legitimacy which enables mobilisation. Moreover, due to the aforementioned widespread uncertainty, more people are susceptible to conspiracy theories ${ }^{5}$. This eases the spread of misinformation or purposefully initiated spread of disinformation which can attract people to specific movements with under false assumptions (CL22) ${ }^{4}$. These processes can be countered by adequate science communication.

\section{Interventions}

In the introduced conceptual model, government responses aim to directly address the spread of epidemic disease or mitigate the negative effects of epidemic upon the society ${ }^{34}$. Through health care related measures such as investments in vaccine development, testing and contact tracing, governments attempt to contain the spread of the epidemic disease (CL23) ${ }^{35}$. A 
specific aspect of health care related response is the public information campaign of (scientific) results to citizens, which is aimed at lowering the widespread uncertainty $(\mathrm{CL} 24)^{4}$. To limit the growth of the epidemic infection rate, governments are forced to establish closure and containment measures to minimise physical contact within the society (CL25) ${ }^{36}$. These restrictions have an immediate effect upon the economy, as recreational activities are limited and people are forced to work from home, moreover people are limited in their freedom (CL26). On the long-term, containment measures can cause severe traumatic psychological effects 5 . By implementation of fiscal, monetary, and financial policy measures governments attempt to mitigate the burden upon the economic stability, state capacity and inequality ${ }^{37}$. Lastly, repression can de-escalate protests in the short-term as the perceived individual price for participation increases (CL27) ${ }^{38}$. However, as repression can lead to escalation of unorganised protests movements which increases the level of grievances (CL28) ${ }^{38}$, the long-term impact of repression can be quite unpredictable ${ }^{8}$.

\section{From grievances, opportunity and mobilisation to civil resistance through data analysis}

The analysis of literature established various drivers for civil resistance movements. The effect of these drivers is fundamentally differentiated by their time-scale variability. For example, a combination of an increase of the infection rate and accompanying strict containment measurements may cause a sudden tension in a society ${ }^{5}$. The socioeconomic effects of pandemic perturb the stability of the society as people require time to adapt to new living conditions. Therefore, the mobilisation of protests can be related to fast changes in the environment.

- H1: The outbreak of protests is related to changes in fast variables.

More specifically within the context of the COVID-19 pandemic, the acceptability of containment measures can be linked to the outbreak of protests. For this purpose, the short-term legitimacy of measures can be calculated as a combined factor of excess deaths and allowed movement of citizens ${ }^{39}$.

- H2: Acceptability of measures is related to the outbreak of protests.

Whereas sudden changes might impose a demanding effort to the adaptive capacity of societies, structural and slowly changing conditions might affect the outbreak of civil resistance in a different kind of way. As such, grievances correlate with outbreaks of civil resistance movements ${ }^{8}$. More people might be receptive to civil resistance movements in countries which have worse structural living conditions. As more people join the protests we can hypothesise that protests in countries with worse living conditions have a relative higher intensity and transform into riots more quickly ${ }^{7}$. As these grievances factors change relatively slowly compared to the sudden changes in the social system environment, it can be generalised that slow variables are better estimators of the intensity of protests.

- H3: Slow variables influence the intensity of the peak of protests

\section{Quantitative and computational Modelling of civil resistance}

The proposed conceptual model includes interactions between socioeconomic conditions, social dynamics and disruptive effects of the pandemic that might facilitate the emergence of civil resistance. In this section, we go a step further in terms of modelling by applying this model to COVID-19 data. For this reason, we link the factors to COVID-19 data proxies to enable quantitative modelling of civil resistance and conduct a cross-national comparison.

Four different information sources are queried to create data proxies. First, global institutions monitor economic and demographic conditions. Second, independent research institutions provide yearly updated reports to monitor global living standards. Third, various data sets are available to analyse the severity of the COVID-19 pandemic, government containment and mitigating strategies at a country level, and changes in behaviour through analysis of mobility. Lastly, the number of protests, riots, and violence against civilians is required to analyse civil resistance movements and repressive reactions by governments. For each of the factors, the temporal resolution of the data should match with the estimated time-scale variability.

Global institutions such as the World Bank and United Nations monitor demography and economic development. The United Nations provides global population statistics at a country level and provides age-cohorts to account for the youth bulge dynamic. The World Bank provides statistics for the percentage of people living in cities. The Organisation for Economic Co-operation and Development (OECD) involving 37 collects measures for (youth) unemployment, GDP and Trust in government, which are of particular interest when comparing the economic and political stability of various countries.

Various independent research institutions monitor the global living conditions in terms of human rights. Freedom House (FH) focusses on human rights. Their 'Freedom in the World' report yields two aggregated indexes for political rights and civil liberties ${ }^{40}$. These indexes map nicely to respectively democracy and freedom. Higher values for these indexes indicate that people experience more freedom, thus score better in terms of human rights. The Fund For Peace measures the stability 


\begin{tabular}{|c|c|c|c|c|c|c|c|}
\hline Factor & Proxy & Category & Pathway & Time-scale & Temporal resolution & Intervention & Data \\
\hline Morbitity due to the disease & Excess mortality & Health care & Dissatisfaction & Intermediate & Weekly & No & Economist \\
\hline Unemployment & Unemployment & Economic & Dissatisfaction & Intermediate & Monthly & No & OECD \\
\hline Group grievance & Group grievance & Social & Dissatisfaction & Slow & Yearly & No & FSI \\
\hline Democracy & Political rights & Political & Opportunity & Slow & Yearly & No & FH \\
\hline Freedom & Civil liberties & Political & Opportunity & Slow & Yearly & No & $\mathrm{FH}$ \\
\hline Economic stability & GDP & Economic & Opportunity & Intermediate & Quarterly & No & OECD \\
\hline Mobility & Mobility data & Social & Opportunity & Quick & Daily & No & Google \\
\hline Government legitimacy & State Legitimacy & Political & Opportunity & Slow & Yearly & No & FSI \\
\hline Closures and containment & Containment measures & Social & Opportunity & Quick & Daily & Yes & OxCGRT \\
\hline Economic stimulus measures & Economic support measures & Economic & Opportunity & Quick & Daily & Yes & OxCGRT \\
\hline Health care response & Health care support response & Health care & Opportunity & Quick & Daily & Yes & OxCGRT \\
\hline Stringency government response & Stringency index & Political & Opportunity & Quick & Daily & Yes & OxCGRT \\
\hline Civil resistance & Protests \& Riots & Social & Mobilisation & Quick & Daily & No & ACLED \\
\hline Youth bulge & Population between 15-24, \% & Social & Mobilisation & Slow & Yearly & No & $\mathrm{UN}$ \\
\hline Urbanisation & Urban population living in cities, \% & Social & Mobilisation & Slow & Yearly & No & World bank \\
\hline Repression & Violence against citizens & Political & Mobilisation & Quick & Daily & Yes & ACLED \\
\hline
\end{tabular}

Table 1. Factors and proxies. Time-scale is an indication of the velocity of change by the proxy indicator. The temporal resolution is the actual scale on which the proxy is measured. Data refers to the data source of the proxy.

of countries in the opposite direction. Their yearly updated Fragile State Index yields various indicators to measure the fragility of the state and deprived living conditions ${ }^{41}$. Their indicators for Uneven Economic Development, Group Grievances, Factionalised Elites, and State Legitimacy provide us with a proxy to analyse differences between countries in terms of economic, social, and political stability. For these indicators, higher scores indicate more fragility.

A number of institutions dedicated their resources to measure the impact of the COVID-19 pandemic. While the Johns Hopkins university provides data for tracking the evolution of the pandemic including positive infections, numerous issues remain with the official tallies ${ }^{42}$. A better estimate to compare the severity of the pandemic in terms of mortality is the number of excess deaths, for which the Economist provides a weakly updated report. With the Oxford COVID-19 Government Response Tracker (OxCGRT), the Blavatnik School of Government systematically collects information on government policy responses to the COVID-19 outbreak ${ }^{35}$. This information is captured by 19 ordinal indicators, of which a higher number indicates a more strict government containment policy, or more economic stimulus. These indicators are aggregated in four indexes, which describe the stringency of the measures, the containment efforts, the health care response, and economic support measures. The indicator for public information campaign is of particular interest to monitor government efforts to reduce widespread uncertainty in the society. Lastly, Community Mobility Reports are provided by Google to enable researchers analysis of the people's changing of behaviour in terms of mobility during the pandemic.

Finally, Armed Conflict Location \& Event Data Project (ACLED) collects disaggregated data for conflict mapping and analysis ${ }^{43}$. The event data is disaggregated by type, location, and actors. The type of the event we interested in this particular project are the protests, riots and violence against citizens to analyse civil resistance, escalation and repression. The type of event is further disaggregated into, for example, protest without intervention and protest with intervention. For protests, ACLED reports the size to account for the participation. However, is merely an indication (e.g. dozens or hundreds) and contains many missing values. ACLED provides a special dataset which contains all events related to the COVID-19 pandemic, which enables to distinguish events for analysis.

The data for civil resistance activity could be matched with all socioeconomic factors for 27 countries (Czechia, Belgium, Slovenia, Spain, Luxembourg, Norway, Germany, Sweden, Netherlands, South Korea, Italy, Greece, United States, Estonia, Denmark, Hungary, Poland, Austria, France, Lithuania, Portugal, Slovakia, Finland, Mexico, Chile, Colombia, Latvia). The events were aggregated on daily and country level scale, which provides a rate of protests and riots per day. The constructed data set provides 7545 days for 27 countries for analysis, which can be described as an (slightly) unbalanced panel data set. Additionally, for approximately $40 \%$ of the protest events, ACLED reports an estimation of the size of the protest in terms of people that participate.

The goal of this modelling effort is to predict the number of (COVID-19 related) protests events for a given day for a given country. Because the protests occurred in a relative short timeframe, we can only differentiate for slow variables through cross-national comparison. While this currently limits the ability to account for longitudinal effects of (very) slow variables, it corresponds with the cross-national differences in the severity and timing of the pandemic in terms of mortality, and various containment and other governmental strategies aimed at mitigating the effects of the pandemic.

In order to explore analyse temporal effects we first plotted the data variables over time. Figure 4 displays the data for Italy with standardised values for the number of COVID-19 related protests per day, the containment measures, the change in movement related to work, and the number of excess deaths. Most notably, the number of protests and the movement seems to oscillate in weekly cycles, which points to a dependency with specific weekdays on which protest and a change of mobility are more likely. More interestingly, the periods of COVID-19 protests are characterised by a fast rise and decay of intensity, while 
keeping a relatively constant level of activity between these periods. Human behaviour often displays a non-Poisson distributed burst character of intense activity followed by no or reduced activity ${ }^{44}$. In order to smooth effect of weekdays, we transform the data of fast variables, such as excess deaths, mobility data and containment and health care measurements, to a rolling average of seven days.
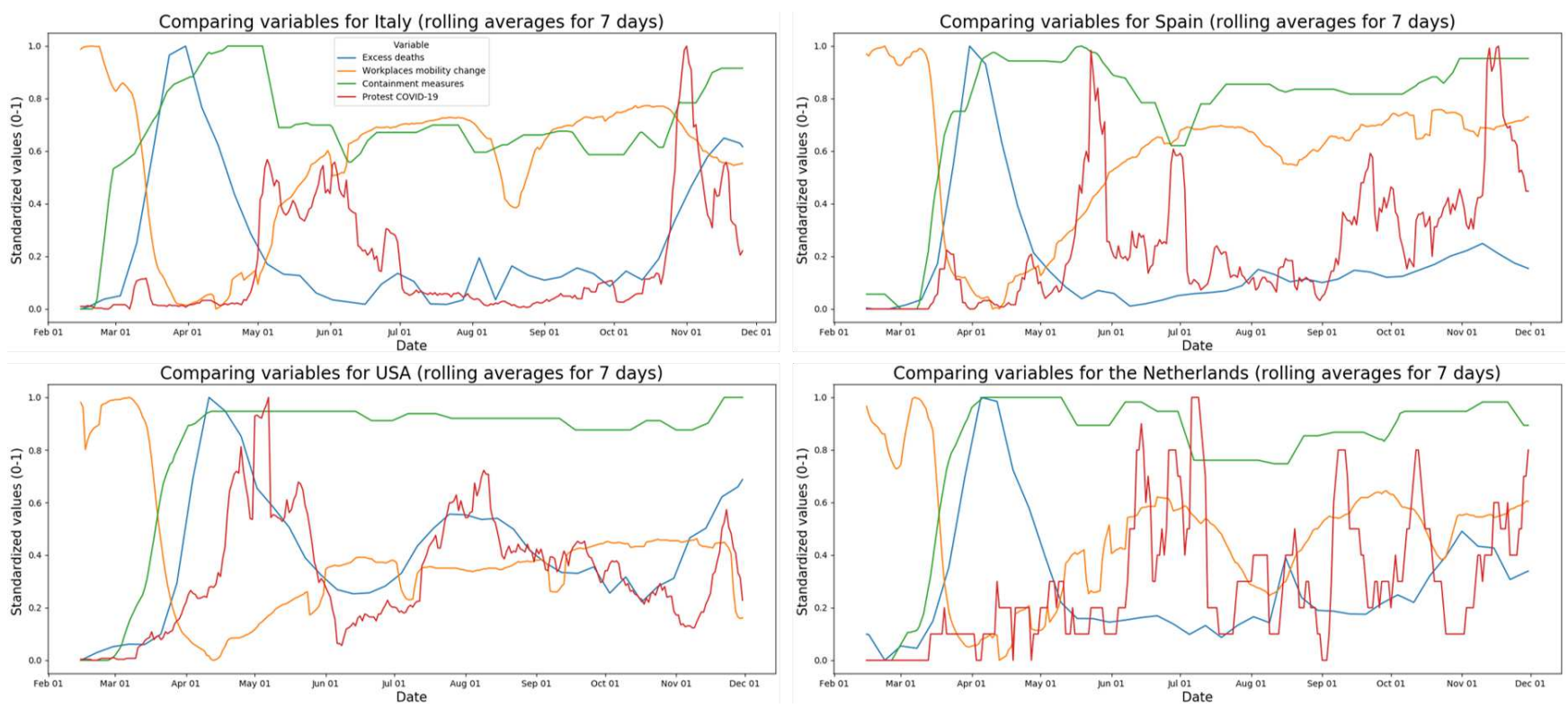

Figure 3. Comparing fast changing variables over time. Values are standardised between 0 and 1.

\section{Regression model}

Exploration of the dependent variable demonstrates an overdispersion of days without protests (Supplementary material). This overdispersion implies that in the observed countries no protest activity takes place in the majority of the period considered, most likely because there is not a particular social tension about COVID-19 related social stress factors. Therefore, we are unable to model the protest behaviour with ordinary count models such as a Poisson- or negative binomial regression model. Instead, we we used a zero-inflated negative binomial regression model to explore the influence of various independent variables, as usually, the negative binomial regression model is better at handling over-dispersion and excessive zeros than the Poisson regression model ${ }^{45}$. We assume that two separate processes determine the number of protests on a particular day. First, the process of social tension assures that people are motivated to engage in civil resistance activities. Without this social tension no civil resistance activities will occur. Second the mobilisation process attracts people towards civil resistance movements and as such the number protests and number of people that participate in the activities yields a data proxy for this variable.

We implement a zero-inflated negative binomial (ZINB) regression model to analyse the number of protests along two steps. First, a logit model is generated to analyse which variables could best predict the absence of protests on specific days. As such the factors contributing to the development process of social tension are analysed. Second, a negative binomial regression model (NBM) is generated to predict the number of protests for days that are not 'certain' zeros. This model analyses which factors contribute to the severity of protests on a specific day. The maximum likelihood estimation (MLE) method is used for optimisation $^{46}$. The Akaike's information criteria (AIC) and log-likelihood are selected as estimators for assessing the performance of the models and model selection ${ }^{45}$.

First, four ZINB models are constructed to compare the predictive value of fast variables. The regression coefficients and AIC scores are summarised and explained in the Supplementary material. The results demonstrate that the model including the lagged variable for the number of protests in the previous week provides the best fit of all individual models. This supports hypothesis 4. Additionally, a model which includes all significant factors provides the best fit overall. In a second step, this ZINB model is compared to a regular NBM model. These results are summarised in the Supplementary material. The AIC values of the model point that a ZINB model provides a better fit. Overall these results show that separate processes are involved in triggering and reinforcing protests events. Finally, the predictive value of slow variables is compared by constructing regular NBM models. As slow variables measured yearly, their temporal resolution prohibits the construction of ZINB models, because it is impossible to differentiate between the logit and NBM model results. The NBM models of slow variables (see Supplementary material), however, provide better fit than the ZINB model of quick variables. This supports hypothesis 3, as the 
slow variables are better estimators of the intensity of protests on a given day.

\section{Analysis of results}

Overall, the log-likelihood of our models suggest that the statistical models provide modest predictive value. Similar to other studies, our data and statistical models are unable to capture all complexity to enable forecasting of conflict ${ }^{47}$. Most likely due to a latent process unobserved by current data or due to the interplay of the different factors that yield unexpected effects. However, it appears that countries with low rates of grievances and high rates of political rights are more likely to experience less severe waves of protests. Contrary to the literature, countries with higher youth bulge are less likely to experience protests, potentially due to less impact by the pandemic. Additionally, the results show that periods without protests are most likely characterised by an absence of a specific process. This could be the tension caused by the effects of containment measures and the mortal impact of the pandemic. As interventions need time to effect a society and people need time to adapt their behaviour to these interventions, other modelling methods are required to take the inherent complexity and temporal dynamics into account.

\section{System dynamics model}

A system dynamics model (SDM) is constructed to further explore the underlying systems temporal behaviour. SDMs can aid in understanding and simulating this system emergent behaviour as they focus on feedback loops and non-linear behaviour of variables in social systems ${ }^{48}$. Therefore, SDMs are characterised as top-down information feedback methods, which do not specify local interactions. By assuming causal relationships between model variables, dynamic behavior can be expressed with difference or differential equations.

As shown in the literature review, civil resistance can emerge in social systems as result of several societal factors like dissatisfaction, opportunity and mobilisation ${ }^{6}$. Using system dynamics modelling, we can model latent processes that yield protests as a function significant factors identified in our statistical models. First, we model the dynamic of people getting dissatisfied and motivated to engage in civil resistance ${ }^{16}$. This generates a pressure on the society. Second, we model the process of mobilisation and disengagement of people in civil resistance movements ${ }^{29}$. This is a self-reinforcing process in which as the system has a high level of tension more people are attracted to the civil resistance movement. These activists than yield protests events that amplify the tension in the system.

The modelling steps are documented according to the Preferred Model Reporting Requirement ${ }^{49}$. We model the system with a set of equations. Each of these equations and their hypothesised effect upon the behaviour are explained. The variables in the SDM (Table 2) are formulated based on the literature based CLD (Figure 2), the constructed data set (Table $1 \&$ Supplementary material), and additional optimising parameters to fit the behaviour of model to the data. After explaining the model, we explore the optimisation results and behavior captured by the model.

\begin{tabular}{|c|c|c|c|c|c|}
\hline Dynamic & Variable & Abbreviation & Dimension & Range & Type \\
\hline \multirow[t]{17}{*}{ Pressure } & Citizens & $\mathrm{C}$ & Persons & \# & Exogenous (Data) \\
\hline & Calm citizens & $\mathrm{CC}$ & Persons & $(0, \mathrm{C})$ & Stock \\
\hline & Potential activists & $\mathrm{CP}$ & Persons & $(0, \mathrm{C})$ & Stock \\
\hline & Social unrest & $\mathrm{CU}$ & Persons / day & $(0, \mathrm{C})$ & Flow \\
\hline & Unemployment & $\mathrm{U}$ & Persons / Persons & $(0,1)$ & Exogenous (Data) \\
\hline & Government legitimacy & LG & Persons / Persons & $(0,1)$ & Exogenous (Data) \\
\hline & Social adaptation & CR & Persons / day & $(0, \mathrm{C})$ & Flow \\
\hline & Freedom & SF & Persons / Persons & $(0,1)$ & Exogenous (Data) \\
\hline & Political Rights & SPR & Persons / Persons & $(0,1)$ & Exogenous (Data) \\
\hline & Pressure velocity & PV & & $(0,1)$ & Optimising \\
\hline & Tension & $\mathrm{T}$ & Persons / (Protest * Persons) & $(0,1)$ & Auxiliary \\
\hline & Tension velocity & TV & - & $(0,1)$ & Optimising \\
\hline & Tension threshold & TT & - & $(0,1)$ & Optimising \\
\hline & Legitimacy of measures & LM & - & $(0,1)$ & Auxiliary \\
\hline & Mobility Residential & MBR & Change from baseline & \# & Exogenous (Data) \\
\hline & Mobility Workplaces & MBW & Change from baseline & $\#$ & Exogenous (Data) \\
\hline & Morbidity due to disease & MR & Change from baseline & $\#$ & Exogenous (Data) \\
\hline \multirow[t]{9}{*}{ Mobilisation } & Protests & $\mathrm{P}$ & Protest & \# & Auxiliary \\
\hline & Protests per citizen & $\mathrm{PC}$ & Protest / citizen & \# & Constant $(0.002 / 1000)$ \\
\hline & Activists & CA & Persons & $(0, \mathrm{CP})$ & Stock \\
\hline & Mobiliation & $\mathrm{CM}$ & Persons / day & $(0, \mathrm{CP})$ & Flow \\
\hline & Disengagement & $\mathrm{CD}$ & Persons / day & $(0, \mathrm{CP})$ & Flow \\
\hline & Youth bulge & $\mathrm{Y}$ & Persons / Persons & $(0,1)$ & Exogenous (Data) \\
\hline & Mobilisation tendency & TM & & $(0,1)$ & Optimising \\
\hline & Mobilisation threshold & MT & - & - & Optimising \\
\hline & Fatigue velocity & $\mathrm{F}$ & - & $(0,10)$ & Optimising \\
\hline
\end{tabular}

Table 2. Description of variables in the SDM. Threshold and velocity variables are included to enable fit the model. 


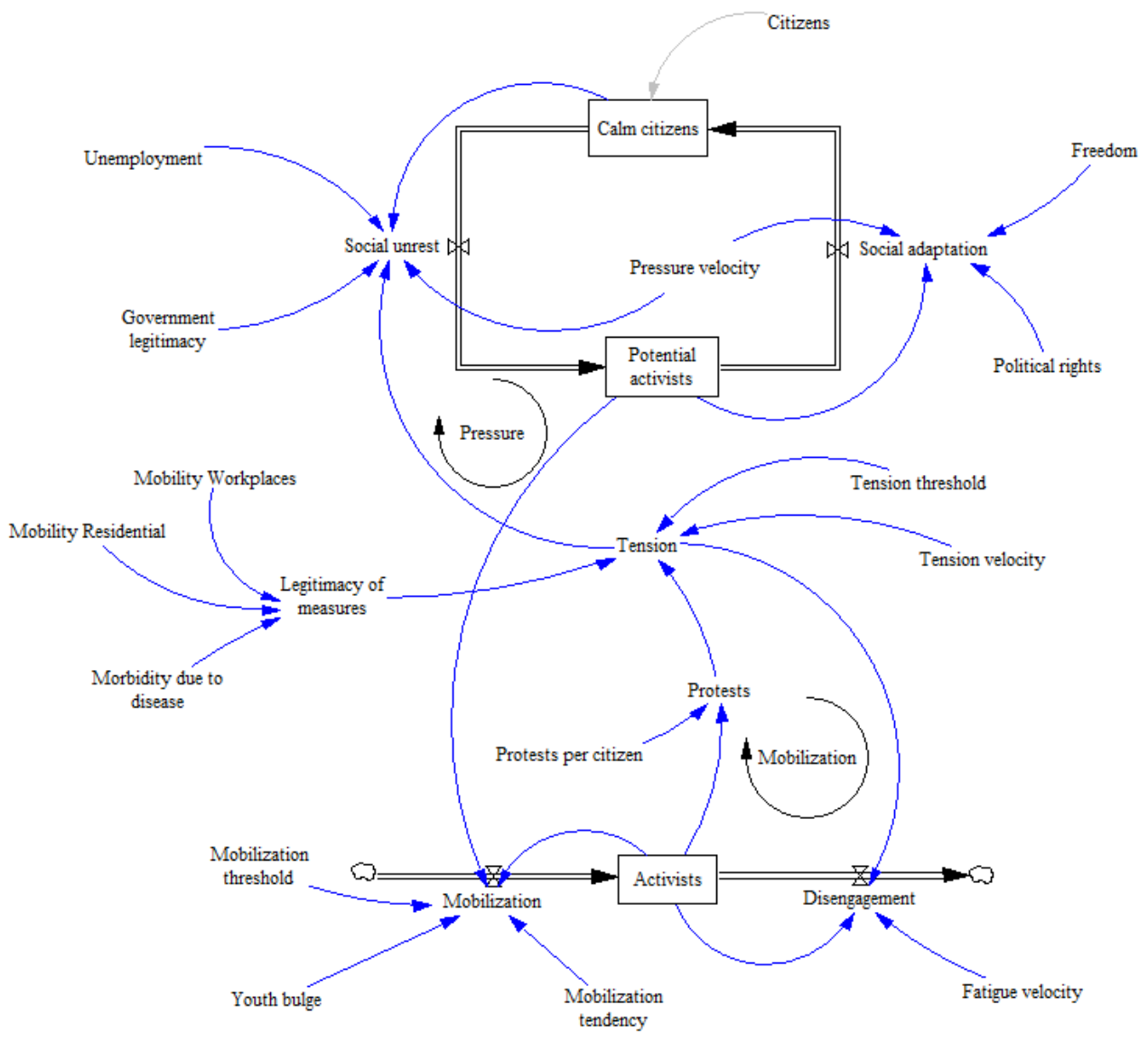

Figure 4. SDM model displaying two processes that explain the emergence of protests. Variables are connected to display causal links. The tension resulting from the legitimacy of measures causes a shock to the system. The system encapsulates two feedback loops. The first feedback loop is the pressure process, as a high level of tension yields a process in which citizens are more susceptible to mobilisation. The second feedback loop is the mobilisation process as a high level of tension as a slowing effect on the disengagement process.

\section{The model equations}

Tension is the central factor in the SDM. A tension (Equation 1) in the model may be triggered as the legitimacy of measurements drops below 1 (Equation 2). As suggested by ${ }^{50}$, we model tension as a sigmoid (Equation 1), in which tension (T) is triggered by a level of legitimacy of measures (LM) below 1 and above the tension threshold value. The parameter for tension velocity controls the slope in which the tension transitions from a relaxed state (0) to a tensed state (1). Furthermore the number of protests $(\mathrm{P})$ amplifies this transition process. Given the nature of the function, the level of the level of tension hovers between 0 and 1 (minimum and maximum tension).

As a trade-off exists between mobility and mortality, for simplicity the level of legitimacy (Equation 2) is modelled as an estimation of the difference between reduced movement and increased mortality in terms of proportionality. In which mortality loosens the reduced legitimacy caused by a reduction of mobility. This is bounded between 0 and 1 (no and full legitimacy).

$$
\begin{aligned}
& T_{t=1}=\frac{1}{1+e^{-(T V+P)\left(\left(1-L M_{t}\right)-T T\right)}} \\
& L M_{t}=\min \left(\max \left(1+M R_{t}+\frac{M B W_{t}+M B R_{t}}{2}, 0\right), 1\right), i f \frac{M B W_{t}+M B R_{t}}{2}<0, \text { else } 1
\end{aligned}
$$

Next, we model the process in which the tension causes citizens to become dissatisfied. Dissatisfied citizens become more susceptible to mobilisation efforts of civil resistance movements and are thus characterised as potential activists. Overall this 
process mimics a pressure valve which controls the pressure upon the society by increasing and decreasing the number of potential recruits for civil resistance movements.

At the start of the simulation, the level of tension is assumed to be zero and thus the total number of calm citizens is assumed to be equal to the population (Equation 3). The flow of citizens from calm citizens to potential activists is calculated by the level of tension amplified by the level of unemployment and reduced by the level of legitimacy of the government (Equations $4 \& 5$ ). The flow from the potential activists to calm citizens is assumed to be steady and accelerated by high levels of freedom and political rights (Equation 6). Furthermore a pressure velocity (PV) is added to calibrate the velocity of the process of people becoming dissatisfied and satisfied.

$$
\begin{aligned}
& C C_{t+1}=C C_{t}-C U t+1+C R_{t+1} \\
& C U_{t+1}=C C_{t+1} \times T_{t+1} \times P V \times \frac{U+L G}{2} \\
& C P_{t+1}=C P_{t}+C U_{t+1}-C R_{t+1} \\
& C R_{t+1}=C P_{t+1} \times P V \times \frac{S P R+S F}{2}
\end{aligned}
$$

Lastly, we model the mobilisation process of civil resistance movements. As a starting point we use the work of ${ }^{51}$. This model applies a population dynamic to model mobilisation in which activists (CA) attract other activists. The increase of the number of activists thus increases as new activists join the movement (Equation 7). As the level of active activists (CA) exceeds the mobilisation threshold (MT) times the number of people in the systems, the increase of activists stabilises at the level of mobilisation tendency (TM). This mobilisation tendency is an optimisation variable, which points to the number of potential activists that are mobilised by each of the current activists. Value above 1, would thus imply exponential growth of the civil resistance movement for relatively low values of activists. As we impute the number of potential activists in the equation, the number of activists increases more rapidly as a high number of people experience aggravation and become sensitive to civil resistance movements. The level of mobilisation is limited between the level of potential activists and the level of activists to secure model consistency.

The mobilisation of a group always starts with 1 , which is why the base level of activists is set at 1 . The mobilisation of activists (CM) is a function of the number of activists (CA) (Equation 8). The disengagement of activists (CD) is modelled as a function of the fatigue velocity and tension, so that disengagement is high when tension is low and disengagement is low when tension is high (Equation 9). The number of protests is a function of the total number of activists (Equation 10), which simply assumes a linear relationship between the number of activists and the number of protests.

$$
\begin{aligned}
& C M_{t+1}=\min \left(\left(T M \times \frac{y b \times C A_{t}}{\left(M T \times\left(C P_{t}+C A_{t}\right)\right)^{2}+C A_{t}^{2}}\right) \times C A_{t},\left(C P_{t}-C A_{t}\right)\right) \\
& C A_{t+1}=\max \left(C A_{t}-C M_{t+1}+C D_{t+1}, 1\right) \\
& C D_{t+1}=e^{-T \times \frac{1}{F} \times C A_{t}} \\
& P_{t}=C A_{t} \times P C
\end{aligned}
$$




\section{Model behaviour and Optimisation results}

The model incorporates six optimisation parameters. The model was implemented in Vensim ${ }^{\mathrm{TM}}$ to explore the behaviour of the model, calibrate the optimising parameters to the data, and test the sensitivity of the model. We selected four countries for the calibration of the model: Italy, Spain, USA, and the Netherlands (see Supplementary material for the payoff function, parameter space and explanation of the optimisation method). These countries were selected as they experienced various protest waves during the year 2020. The time-step in the model was set to one day to match the scope of the data of the exogenous variables that are linked to the tension equation and pressure dynamic.

Whilst optimisation of the model converged for the Netherlands and Spain, the model provided a poor fit for Italy and Spain data (See Supplementary material). Various explanations for these differences exist. For example, the impact of the pandemic can be different from one country to another. As the tension factor in our model is based on mobility and excess deaths, these conditions could provide good explanation for the protests in Spain and the Netherlands and worse for the other two countries. Moreover, due to the size of the USA, underlying reasons for protests could differ between states in the US. This could point to a specific unidentified maximum spatial scope for analysing this phenomenon. In comparison to pure statistical approaches, the approach of SDM enables to analyse specific dynamics explicitly. The explicit modelling of temporal effects such as tension, recruitment, and disengagement enables to better understand temporal dependencies between socioeconomic conditions, interventions, and the emergent patterns.

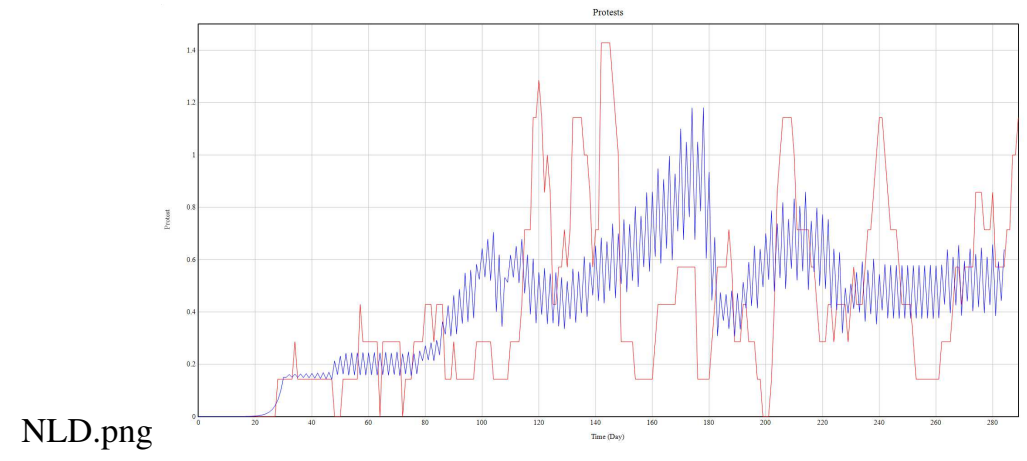

Figure 5. Fit of the model to the data for the Netherlands. Red line is a 7 day rolling average for the actual number of protests, the blue line is the simulated data for the optimised parameter setting. (See Supplementary material for full parameter setting).

\section{Discussion}

In this paper we have presented a holistic analysis approach of the emergence of civil resistance during the outbreak of the COVID-19 pandemic. For this analysis, we first combined a qualitative literature based approach to identify relevant factors related to both the emergence of civil resistance in general and the disruptive effect of the pandemic upon the society. The resulting causal-loop diagram (CLD) provides a conceptual understanding that brings together the different scientific perspectives for the first time. Secondly, from open sources we construct a data set of factors that include socioeconomic conditions and societal dynamics. We use this data set to explore whether we can empirically identify correlates between these factors and the outbreak of protests. For this analysis we compared various statistical models in terms of their fit. Our outcomes suggests that while fast variables have a better explanatory value of 'when' protests will happen, slow variables are better estimates of the 'how' in terms of the relative intensity of the protests. Additionally, the data analysis demonstrates there is a strong need for other modelling approaches that better capture the complexity and underlying dynamics. As such, we have developed a system dynamics model (SDM) based on the significant factors identified in our statistical models. In our model we make literature based assumptions about underlying dynamics which are effected by the incorporated factors. With this model, we are able to analyse the hypothesised interplay between the development of pressure in a society and mobilisation of civil resistance, enabling analysis of various feedback loops. The analysis of our SDM model demonstrates that our model might provide a good explanation of the underlying dynamics of the protests in the Netherlands and Spain, but less explanation for the protests in the USA and Italy.

This paper builds upon promising efforts that attempt to quantify the potential of an outbreak of conflicts for specific countries or regions ${ }^{8,9}$. Our holistic approach demonstrates that future models would improve their precision as they account for the complexity of the underlying dynamics. While the COVID-19 pandemic has a severe and disruptive effect upon the society at large, it also provides a unique window to study the emergence of civil resistance and these underlying social dynamics. The effect of several containment strategies has had a disruptive effect upon the behaviour of people, which has triggered the 
mobilisation of civil resistance movements. Our analysis suggests that while capturing these fast changing disruptions might improve our understanding of when civil resistance might happen, slowly developments in structural conditions might still be better estimates of the severity of such outbreaks.

Our modelling efforts are limited by some practical and theoretical constrains. In general, selecting relevant factors and extracting associated data to model the tension is hard. Especially, the identification of relevant factors that capture the emergence of political opportunity is hard due to changing perceptions. The trigger for civil resistance in our model was limited to changes in mobility, impact of the pandemic, and socioeconomic conditions. Due to limited information about the underlying motivations for the protests, we were not able to discriminate between them. For example, this could explain the poor fit of our SDM for the USA and Italy, in which potentially protests were motivated differently. Capturing other issues, such as current legislation related to vaccination, could potentially further explain existing tension. We excluded the containment interventions from our SDM, as analysis of the effectiveness of the interventions was beyond the scope of this project.

Additionally, our analysis was limited to the year 2020 as we rely on the ACLED data set for daily protest data. Most of the countries in our data set are only available at ACLED for this particular year. Moreover, the importance of the Black-LivesMatter movement in this given year could have a interfering effect upon both civil resistance movements and media coverage. Bias in news based event databases is well known ${ }^{52}$. Future modelling efforts would profit from such detailed data sets over multiple years, as it would enable to analyse the effect of changes in slowly developing structural socioeconomic conditions upon the emergence of civil resistance within countries rather than between countries.

The predictive power of models would possibly profit from several improvements in the granularity and quality of the data. For example, better global estimates of the number of attendees of protests would enable to better fit the mobilisation process of our SDM. While development of such data sets are taking place at ACLED and CCC, the current progress, quality, or scope of such data sets is insufficient to optimise models ${ }^{43,53}$. Combining other modelling approaches could be another way to improve predictive power. Two other modelling suggestions for modelling we identified along this project. First, while our modelling approaches accounts for the change of behaviour in terms of mobility more in-depth analysis of individual behaviour would enable to better explain the burstiness of protests. For example, modelling the contagion of behavior using agent-based modelling would enable to better capture the gathering of people into protests. However, these models require fine-grained data sets or detailed stylised facts of these dynamics. Sentiment analysis of social media outlets such as Twitter would enable to estimate the effect of emotional change or social contagion in a society. Secondly, news coverage on civil resistance movements and societal conditions, or the spread of disinformation could influence the way people perceive their environment and living conditions. Accounting these dynamics would enable us to better understand the driving dynamics of civil resistance movement and underlying motivations for mobilisations, which would allow policy makers and human rights institutions to anticipate upon societal instability and improve social cohesion.

\section{References}

1. Amenta, E., Caren, N., Chiarello, E. \& Su, Y. The political consequences of social movements. Annu. Rev. Sociol. 36, 287-307 (2010).

2. Carothers, T. \& O’Donohue, A. Polarization and the pandemic (2020).

3. Madhav, N. et al. Pandemics: Risks, impacts, and mitigation. Dis. Control. Priorities: Improv. Heal. Reducing Poverty (2017).

4. Van Bavel, J. J., Baicker, K., Boggio, P. S. \& Capraro, V. Using social and behavioural science to support covid-19 pandemic response. Nat. Hum. Behav. 4, 460-471 (2020).

5. Taylor, S. The psychology of pandemics: Preparing for the next global outbreak of infectious disease (Cambridge Scholars Publishing, 2019).

6. Gallo, G. Conflict theory, complexity and systems approach. Syst. Res. Behav. Sci. 30, 156-175 (2013).

7. Goldstone, J. A. et al. A global model for forecasting political instability. Am. J. Polit. Sci. 54, 190-208 (2010).

8. Chenoweth, E. \& Ulfelder, J. Can structural conditions explain the onset of nonviolent uprisings? J. Confl. Resolut. 61, 298-324 (2017).

9. Hegre, H. et al. Views: a political violence early-warning system. J. peace research 56, 155-174 (2019).

10. Turchin, P., Gavrilets, S. \& Goldstone, J. A. Linking "micro" to "macro" models of state breakdown to improve methods for political forecasting. Cliodynamics $\mathbf{8}$ (2017).

11. Hastings, A. et al. Transient phenomena in ecology. Science 361 (2018).

12. Flack, J. C. Multiple time-scales and the developmental dynamics of social systems. Philos. Transactions Royal Soc. B: Biol. Sci. 367, 1802-1810 (2012). 
13. Saxton, G. D. Repression, grievances, mobilization, and rebellion: A new test of gurr's model of ethnopolitical rebellion. Int. Interactions 31, 87-116 (2005).

14. Stephan, M. J. \& Chenoweth, E. Why civil resistance works: The strategic logic of nonviolent conflict. Int. security 33, 7-44 (2008).

15. Gurr, T. R. Why minorities rebel: A global analysis of communal mobilization and conflict since 1945. Int. Polit. Sci. Rev. 14, 161-201 (1993).

16. Cederman, L.-E., Wimmer, A. \& Min, B. Why do ethnic groups rebel? new data and analysis. World politics 62, 87-119 (2010).

17. Fearon, J. D. \& Laitin, D. D. Ethnicity, insurgency, and civil war. Am. political science review 97, 75-90 (2003).

18. Meyer, D. S. \& Minkoff, D. C. Conceptualizing political opportunity. Soc. forces 82, 1457-1492 (2004).

19. Goldstone, J. A. Ideology, cultural frameworks, and the process of revolution. Theory Soc. 20, 405-453 (1991).

20. Kenrick, D. T., Griskevicius, V., Neuberg, S. L. \& Schaller, M. Renovating the pyramid of needs: Contemporary extensions built upon ancient foundations. Perspectives on psychological science 5, 292-314 (2010).

21. Kapiriri, L. \& Ross, A. The politics of disease epidemics: a comparative analysis of the sars, zika, and ebola outbreaks. Glob. Soc. Welf. 7, 33-45 (2020).

22. Bell, C. \& Lewis, M. The economic implications of epidemics old and new. SSRN Electron. J. (2005).

23. Jonung, L. \& Roeger, W. The macroeconomic effects of a pandemic in europe. a model-based assessment. Eur. Econ. papers (2006).

24. Person, B. et al. Fear and stigma: the epidemic within the sars outbreak. Emerg. infectious diseases 10, 358 (2004).

25. Engels, B. Nothing will be as before: Shifting political opportunity structures in protests against gold mining in burkina faso. The Extr. Ind. Soc. 5, 354-362 (2018).

26. Groeniger, J. O., Noordzij, K., van der Waal, J. \& de Koster, W. Dutch covid-19 lockdown measures increased trust in government and trust in science: A difference-in-differences analysis. Soc. Sci. \& Medicine 275, 113819 (2021).

27. Brooks, S. K. et al. The psychological impact of quarantine and how to reduce it: rapid review of the evidence. The lancet 395, 912-920 (2020).

28. Schroeter, R., Jovanovic, A. \& Renn, O. Social unrest: A systemic risk perspective. Planet@ Risk 2 (2014).

29. Van Stekelenburg, J. \& Klandermans, B. The social psychology of protest. Curr. Sociol. 61, 886-905 (2013).

30. González-Bailón, S., Borge-Holthoefer, J., Rivero, A. \& Moreno, Y. The dynamics of protest recruitment through an online network. Sci. reports 1, 1-7 (2011).

31. Kelly, J. R., Iannone, N. E. \& McCarty, M. K. Emotional contagion of anger is automatic: An evolutionary explanation. Br. J. Soc. Psychol. 55, 182-191 (2016).

32. Huremović, D. Psychiatry of pandemics: a mental health response to infection outbreak (Springer, 2019).

33. Espinola, M. et al. Fear-related behaviors in situations of mass threat. Disaster health 3, 102-111 (2016).

34. Greer, S. L., King, E. J., da Fonseca, E. M. \& Peralta-Santos, A. The comparative politics of covid-19: The need to understand government responses. Glob. public health 15, 1413-1416 (2020).

35. Hale, T., Petherick, A., Phillips, T. \& Webster, S. Variation in government responses to covid-19. Blavatnik school government working paper 31, 2020-11 (2020).

36. Deb, P., Furceri, D., Ostry, J. D. \& Tawk, N. The effect of containment measures on the covid-19 pandemic. CEPR Covid Econ. (2020).

37. Elgin, C., Basbug, G. \& Yalaman, A. Economic policy responses to a pandemic: Developing the covid-19 economic stimulus index. Covid Econ. 1, 40-53 (2020).

38. Larsen, V. B. Organization, Repression, and the Violent Escalation and De-Escalation of Nonviolent Protest. Peace Research Institute Oslo (PRIO) (2020).

39. Guglielmi, S. et al. Public acceptability of containment measures during the covid-19 pandemic in italy: how institutional confidence and specific political support matter. Int. J. Sociol. Soc. Policy (2020).

40. Repucci, S. A leaderless struggle for democracy. Free. House 2020-02 (2020). 
41. Messner de Latour, J. et al. Annual report 2020. Fragile States Index 2020 (2020).

42. Weinberger, D. M. et al. Estimation of excess deaths associated with the covid-19 pandemic in the united states, march to may 2020. JAMA Intern. Medicine 180, 1336-1344 (2020).

43. Raleigh, C., Linke, A., Hegre, H. \& Karlsen, J. Introducing acled: an armed conflict location and event dataset: special data feature. J. peace research 47, 651-660 (2010).

44. Barabasi, A.-L. The origin of bursts and heavy tails in human dynamics. Nature 435, 207-211 (2005).

45. Hilbe, J. M. Negative binomial regression (Cambridge University Press, 2011).

46. Cameron, A. C. \& Trivedi, P. K. Regression analysis of count data, vol. 53 (Cambridge university press, 2013).

47. Cederman, L.-E. \& Weidmann, N. B. Predicting armed conflict: Time to adjust our expectations? Science 355, 474-476 (2017).

48. Sterman, J. D. System dynamics modeling: tools for learning in a complex world. California management review 43, 8-25 (2001).

49. Rahmandad, H. \& Sterman, J. D. Reporting guidelines for simulation-based research in social sciences. Wiley-Blackwell Pubishers (2012).

50. Berestycki, H., Nadal, J.-P. \& Rodíguez, N. A model of riots dynamics: Shocks, diffusion and thresholds. Networks \& Heterog. Media 10, 443 (2015).

51. Morozov, A., Petrovskii, S. \& Gavrilets, S. The yellow vests movement-a case of long transient dynamics? . (2019).

52. Mahoney, C. W. More data, new problems: Audiences, ahistoricity, and selection bias in terrorism and insurgency research (2018).

53. Phillips, P. J. \& Pohl, G. Crowd counting: a behavioural economics perspective. Qual. \& Quant. 1-18 (2021). 


\section{Supplementary Files}

This is a list of supplementary files associated with this preprint. Click to download.

- SupplementaryInformation.pdf 